\title{
Epidemiologia das Doenças Cerebrovasculares em Diamantina, 1998 - 2006
}

\author{
Epidemiology of Cerebrovascular Disease In Diamantina City, 1998 - 2006
}

\author{
Hércules Ribeiro Leite', Ana Paula Nogueira Nunes², José Márcio Mucida \\ Couto ${ }^{3}$, Débora Alves de Ávila Bueno Leite ${ }^{3}$, Paula Aryane Brito Alves ${ }^{4}$
}

\section{RESUMO}

As doenças cardiovasculares acarretam taxas de mortalidade proporcionais a 32,3\%, constituindo as principais causas de óbito. Este grupo é liderado pela doença cerebrovascular (DCV), responsável por um terço das mortes. Objetivo. Analisar alguns aspectos epidemiológicos da DCV através da elaboração de coeficientes de mortalidade. Método. Realizou-se um estudo ecológico de séries temporais para intervalo de 1998 a 2006, n o município de Diamantina-MG. Resultados. Os resultados mostraram coeficientes de mortalidade por DCV em Diamantina com valores superiores quando comparados a outros do estado de Minas Gerais na maioria dos anos. Quanto ao gênero, entre os anos de 1998 a 2006 houve um maior número de óbitos em homens (340,7/ 100.000 habitantes) em relação às mulheres (289,3/100.000 habitantes), maior incidência de óbitos com o avançar da idade em ambos os gêneros, sendo maior em Diamantina (12.750,4/100.000 habitantes) do que no restante do estado de Minas Gerais (9.088,2/100.000 habitantes). Entretanto, a Razão de Mortalidade Proporcional apresentou: 30-39 (16,67\%), 40-49 (17,12\%), 50-59 (18,03\%), 60-69 (17,44\%), 70-79 (17,65\%) e 80/mais anos $(12,44 \%)$. Conclusáo. Dessa forma, são necessários estudos que objetivam identificar os fatores associados à mortalidade para que, num segundo momento, possa planejar e implementar políticas de saúde que visem reduzir essa mortalidade.

Unitermos. Acidente Vascular Encefálico, Mortalidade, Epidemiologia.

Citaçáo. Leite HR, Nunes APN, Couto JMM, Leite DAÁB, Alves $\mathrm{PAB}$. Epidemiologia das Doenças Cerebrovasculares em Diamantina, 1998 - 2006.

\begin{abstract}
The cardiovascular disease cause mortality rates proportional to $32,3 \%$, constituting the main causes of death in Brazil. This group is led by Cerebrovascular Disease (CVD), represents the third cause of deaths. Objective. The aim was to analyze some epidemiological aspects of CVD through development of mortality rates. Method. We performed an ecological study of temporal series to from 1998 to 2006, in Diamantina city, MG. Results. The results showed mortality rates by CVD in Diamantina with higher values when compared to other state of Minas Gerais in most years analyzed. We found that between the years 1998 to 2006 there was a higher number of death in men (340,7/ 100.000 inhabitants) than women (289,3/ 100.000 inhabitants), higher incidence of deaths with the increase of age in both genders, but higher in Diamantina (12.750,4/ 100.000) than in the rest of the state of Minas Gerais (9.088,2/ 100.000 inhabitants). However, the Rate of Proportional Mortality showed: 30-39 (16,67\%), 40-49 (17,12\%), 50-59 (18,03\%), 60-69 (17,44\%), 70$79(17,65 \%)$ e 80 years old and more $(12,44 \%)$. Conclusion. Thus, further studies are needed to identify factors associated with mortality in a second time, to plan and implement health policies that reduce this mortality.
\end{abstract}

Keywords. Stroke, Mortality, Epidemiology.

Citation. Leite HR, Nunes APN, Couto JMM, Leite DAAB, Alves PAB. Epidemiology of Cerebrovascular Disease In Diamantina City, 1998 - 2006.
Trabalho realizado na Universidade Federal dos Vales do Jequitinhonha e Mucuri - UFVJM, Diamantina-MG, Brasil.

1.Fisioterapeuta, Professor Adjunto da Universidade Federal do Amazonas (UFAM), Coari-AM, Brasil.

2.Fisioterapeuta, Mestre em Saúde Pública pela Universidade Federal de Minas Gerais - UFMG, Belo Horizonte-MG, Brasil.

3.Fisioterapeuta, Especialista em Acupuntura, Professora da Universidade Federal do Amazonas, Coari-AM, Brasil.

4.Médica Veterinária, Professora Adjunta da Universidade Federal dos Vales do Jequitinhonha e Mucuri (UFVJM), Diamantina-MG, Brasil.
Endereço para correspondência: Paula Aryane Brito

Campus JK - Rodovia MGT 367 - Km 583 Universidade Federal dos Vales do Jequitinhonha e Mucuri (UFVJM) E-mail: pbrito@ufvjm.edu.br 


\section{INTRODUÇÃO}

Segundo o Ministério da Saúde (2000), o Brasil, desde a década de 1940, vem passando por um processo de inversão das curvas de mortalidade, observando-se um declínio de mortes por doenças infecciosas e um concomitante aumento por doenças crônicas não-transmissíveis e causas externas ${ }^{1}$. É nesse contexto que as doenças do aparelho circulatório adquirem relevância no perfil de morbi-mortalidade do país ${ }^{2}$. Este grupo tem como representante principal a Doença Cerebrovascular (DCV), sendo responsável por um terço dos óbitos ${ }^{3}$.

Segundo a Organização Mundial de Saúde (OMS), a doença cerebrovascular foi considerada a segunda causa de mortalidade no mundo em 1990 e a terceira causa de mortalidade em países desenvolvidos, sendo assim, responsável por aproximadamente 4,4 milhóes de mortes no mundo. Em 1996, o índice de mortalidade por DCV foi de 56,1 por 100000 habitantes $^{4}$. Estatísticas mais recentes indicam que a DCV determinou 5,54 milhōes de óbitos no mundo, em 1999, com dois terços destes ocorrendo em países menos desenvolvidos 5 . Além de contribuir para a mortalidade e morbidade, essa doença também implica em altos custos ${ }^{6}$.

Devido às mudanças no estilo de vida, acredita-se que a DCV continuará constituindo um problema de saúde pública de relevância nesse século na população com idade avançada ${ }^{3}$. A DCV pode ocorrer em indivíduos jovens sendo relacionada a fatores de risco como os distúrbios da coagulação, as doenças inflamatórias e imunológicas, bem como ao uso de drogas ${ }^{7}$.

No Brasil, a mortalidade por DCV é bastante alta, sendo que em 1985 ocorreram 73.205 óbitos por estas afecções $\left(9,3 \%\right.$ do total dos óbitos) ${ }^{4}$, acometendo especialmente maior porcentagem de homens quando comparadas em mulheres na cidade de Belo Horizonte (MG), bem como no estado de Minas Gerais ${ }^{8}$.

No município de Diamantina há uma carência de estudos epidemiológicos que possam esclarecer a ocorrência da doença e consequentemente subsidiar programas de prevenção e controle da mesma, além da ausência de fisioterapeutas nas unidades de Estratégia de Saúde da Família (ESF's). A fisioterapia é essencial no tratamento da fase aguda e crônica do acidente vascular cerebral, mas o seu papel é limitado na prevenção e controle desta doen- ça. É importante ressaltar a necessidade de um acompanhamento fisioterápico em longo prazo dos sobreviventes, tanto no trabalho de prevenção de complicaçóes como na neuroreabilitação como um todo, incluindo a terapia ocupacional. Assim, o objetivo do presente trabalho é analisar alguns aspectos epidemiológicos da DCV em Diamantina no intervalo cronológico de 1998 a 2006, através da elaboração de coeficientes de mortalidade.

\section{MÉTODO}

Foi realizado um estudo ecológico de séries temporais para intervalo cronológico de 1998 a 2006, tendo como base territorial o município de Diamantina em Minas Gerais. Para tanto foram calculados os seguintes indicadores de saúde: coeficiente de mortalidade por DCV (no óbitos por DCV/ população total x 100.000 habitantes), coeficiente de mortalidade específico por idade ( $\mathrm{n}^{\circ}$ óbitos por DCV em determinada faixa etária/população total de determinada faixa etária x 100.000 habitantes), coeficiente de mortalidade específico por gênero para a DCV (no óbitos por DCV em determinado gênero/população total de determinado gênero x 100.000), Razão por Mortalidade Proporcional (RMP) por idade ( $\mathrm{n}^{\circ}$ óbitos por DCV em determinada faixa etária/ total de óbitos na respectiva faixa etária x 100) e RMP por gênero na população com 80 anos e mais ( $n^{\circ}$ óbitos por DCV no gênero na faixa etária de 80 anos e mais/total de óbitos no gênero na respectiva faixa etária x 100).

Para a construção dos coeficientes de mortalidade, foram utilizados os dados do Sistema de Informação sobre Mortalidade do Ministério da Saúde (SIM/SMS) ${ }^{9}$ sobre os óbitos dos residentes de Diamantina e de Minas Gerais, analisados no período de 1998 a 2006. A análise temporal se restringiu até 2006 , pois foi o último ano de disponibilidade dos dados no sistema consultado no período de análise dos coeficientes.

Os coeficientes de mortalidade para cada 100.000 habitantes foram calculados considerando-se a populaçáo residente em Diamantina em cada ano. Os dados populacionais foram obtidos a partir do censo de 2000, e a contagem populacional de 1996 do Instituto Brasileiro de Geografia e Estatística (IBGE). Para os anos intercensitários foram utilizadas estimativas populacionais do Ministério da Saúde, disponibilizados na internet através do DATASUS. 
Para a coleta dos dados referentes aos óbitos, foi utilizado o capítulo IX (doenças do aparelho circulatório) da última Classificação Internacional de Doenças (CID-10), e foram coletados os dados referentes às seguintes subclassificaçôes das DCV's: acidente vascular isquêmico transitório e síndromes correlacionadas, hemorragia intracraniana, infarto cerebral, acidente vascular não específico a hemorrágico ou isquêmico e outras doenças cerebrovasculares.

Após a elaboração dos coeficientes, foi realizada uma análise exploratória dos mesmos.

\section{RESULTADOS}

No presente estudo, o coeficiente de mortalidade por DCV de 317,9/100.000 habitantes no município de Diamantina no período de 1998 a 2006, enquanto em Minas Gerais o coeficiente foi de 217,9/100.000 habitantes no mesmo período. $\mathrm{O}$ resultado da análise dos coeficientes específicos de mortalidade por este grupo de causas encontra-se no Gráfico 1. Observou-se um aumento da mortalidade por DCV em Diamantina nos anos de 2000, 2003 e 2006 (52/100.000; 49,7/100.000; $52 / 100.000$ habitantes, respectivamente). Em Minas Gerais, encontrou-se uma tendência à estabilidade dos coeficientes de óbitos por DCV durante o período analisado.

Em relação ao gênero, o masculino apresentou taxas de mortalidade mais elevadas nos anos de 2000, 2003 e $2006(33,9 / 100.000 ; 27,1 / 100.000 ; 33,9 / 100.000$ habitantes; respectivamente). $\mathrm{O}$ gênero feminino não apresentou grandes variaçóes nos coeficientes de mortalidade entre 1998 e 2006 (Gráfico 2). Em Minas Gerais, encontrou-se índices superiores no gênero masculino durante todo o período analisado (Gráfico 3), e observou-se tendência à estabilidade nos coeficientes de mortalidade nos dois gêneros.

Quanto à faixa etária, encontrou-se coeficientes de mortalidade por DCV mais elevados em todas as faixas etárias, em especial na faixa etária acima dos 80 anos de idade, sendo 5686,5/100.000 habitantes na população masculina e 4413,4/100.000 habitantes na feminina em Diamantina (Gráfico 4a). Em Minas Gerais, foi evidenciada a mesma tendência, observando-se 477,4/100.000 habitantes na população masculina e 433,4/100.000 habitantes na feminina na faixa etária de 80 anos ou mais (Gráfico 4b). Em Diamantina, observou-se que as taxas foram maiores na população masculina em relação à feminina nas faixas etárias acima dos 40 anos de idade (Gráfico 4a). Em Minas Gerais, os coeficientes foram superiores na população masculina em todas as faixas etárias analisadas (Gráfico 4b).

Ao analisar a Razão por Mortalidade Proporcional (RMP) por DCV no município de Diamantina entre 1998 e 2006, observou-se que a doença, determina um percentual significativo de óbitos em todas as faixas etárias analisadas (Tabela 1). Quanto à RMP por gênero na faixa etária de 80 anos e mais, encontrou-se $15 \%$ na populaçáo masculina, e $10,95 \%$ na feminina.

\section{DISCUSSÃO}

Observou-se neste estudo, coeficientes de mortalidade por DCV em Diamantina com valores superiores quando comparados a Minas Gerais na maioria dos anos analisados. A DCV é uma patologia de elevada prevalência determinando sérias consequências médicas e sociais, como sequelas de ordem física, de comunicação, funcionais, emocionais, além de constituir uma das principais causas de óbito no Brasil ${ }^{10}$. A mortalidade pela DCV éainda elevada no Brasil quando comparada a outros países ${ }^{11}$.

Quanto ao gênero, houve um maior número de óbitos em homens (340,7/100.000 habitantes) em relação às mulheres (289,3/100.000 habitantes). Este resultado está de acordo com o estudo realizado em Belo Horizonte (MG), onde 39,3\% dos óbitos eram femininos e $60,7 \%$ masculinos $^{13}$. Em Minas Gerais, os valores no gênero masculino mostraram-se superiores durante todo o período analisado, o mesmo aconteceu no estado de São Paulo ${ }^{11,12}$. A maior prevalência masculina poderia ser explicada por fatores como: maus hábitos de vida (tabagismo, etilismo, dieta inadequada e sedentarismo), ausência de prevenção, bem como resistência ao diagnóstico de patologias que predispóem as DCV's' ${ }^{12}$. Os coeficientes do gênero masculino mostraram-se maiores em Diamantina quando comparados a $\mathrm{MG}$ e com picos nos anos de 2000, 2003 e $2006^{12}$.

Os coeficientes do gênero feminino, além de menores, mantiveram-se constantes ao longo dos anos em Diamantina e em Minas Gerais comparados aos masculinos. Não foi observado o mesmo resultado num estudo que teve como base a população brasileira ${ }^{13}$. 


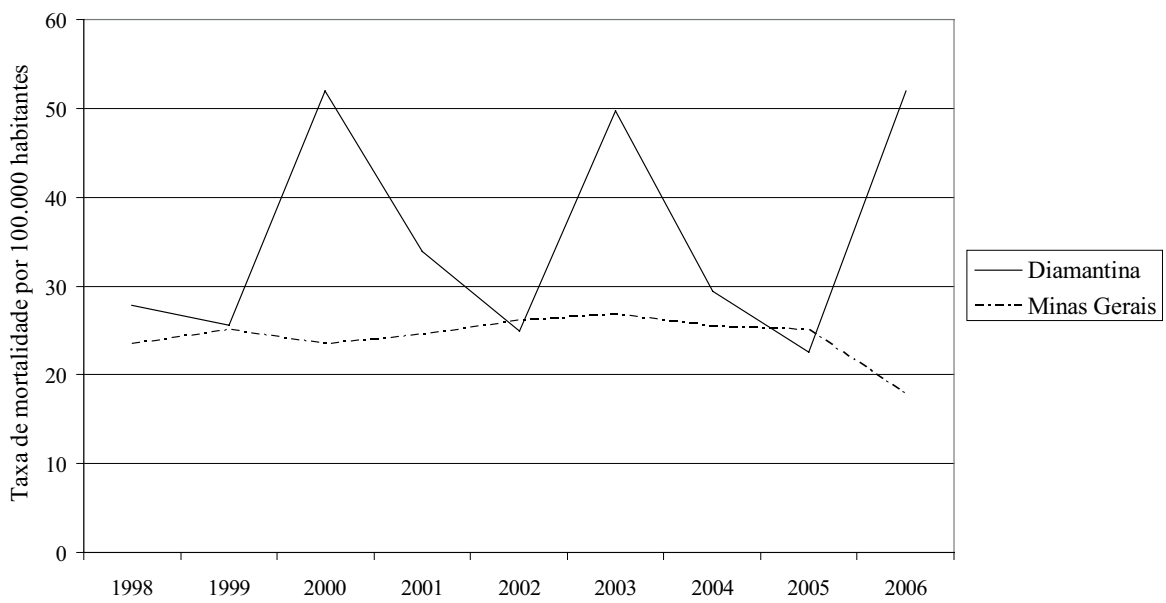

Gráfico 1. Coeficientes de Mortalidade por DCV em Diamantina e Minas Gerais, entre 1998-2006. Fonte: DATASUS (2007)

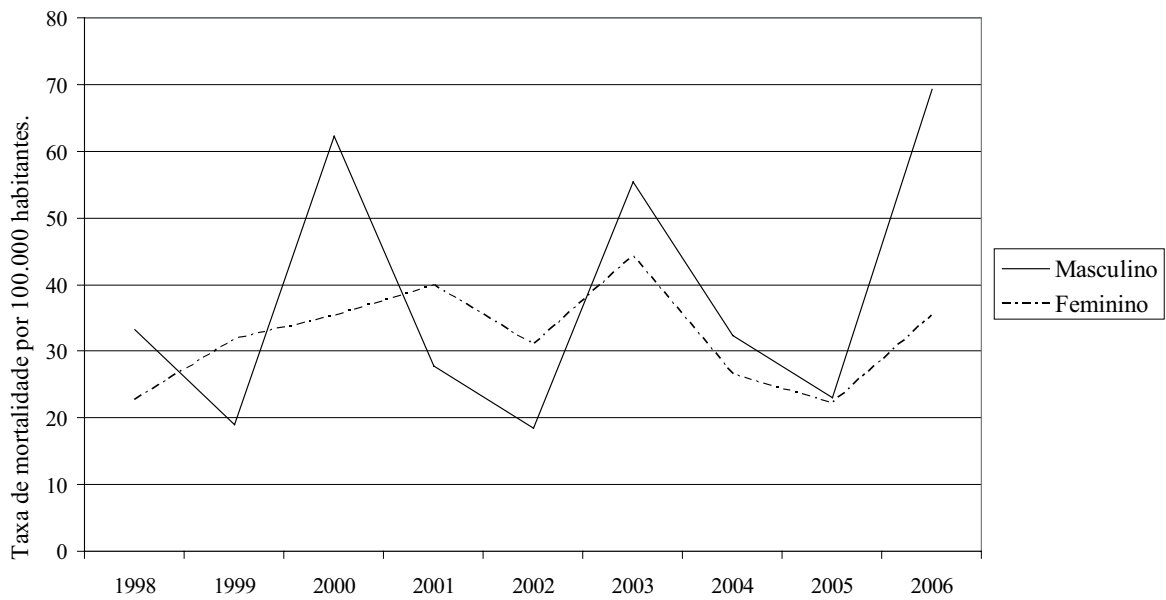

Gráfico 2. Coeficiente de Mortalidade por gênero em Diamantina, entre 1998-2006. Fonte: DATASUS (2007)

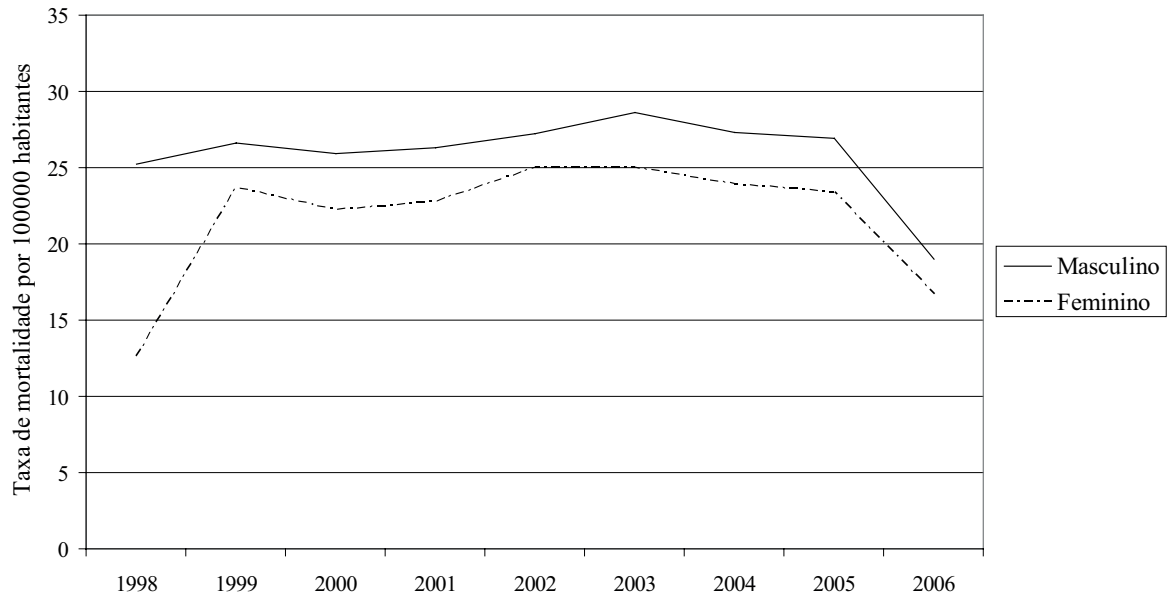

Gráfico 3. Coeficiente de Mortalidade por gênero em Minas Gerais, entre 1998-2006. Fonte: DATASUS (2007) 


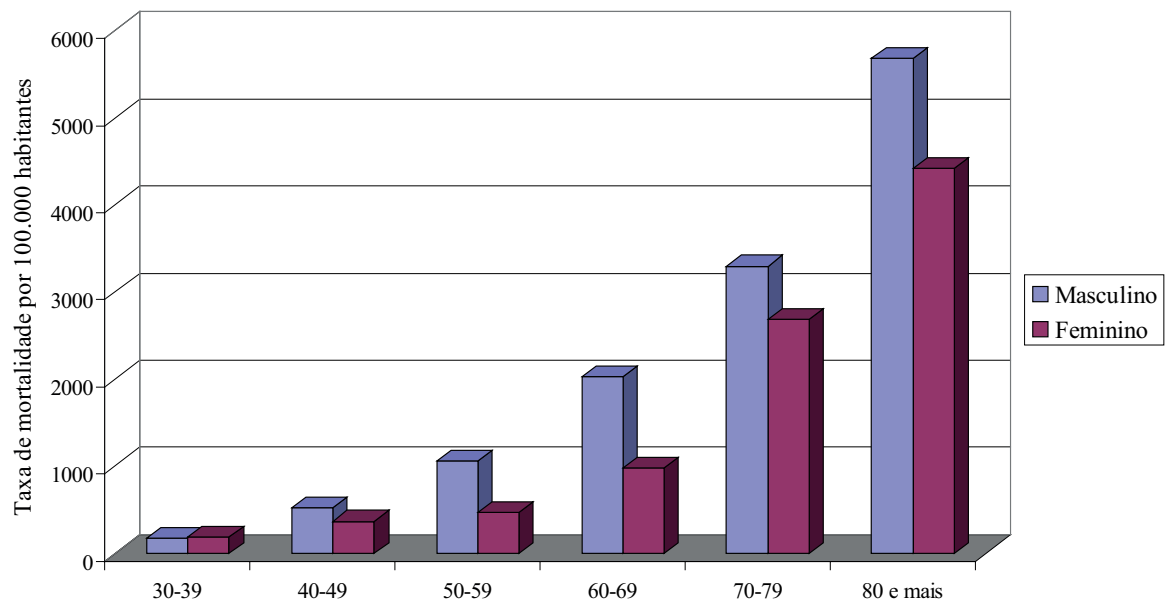

Gráfico 4a. Coeficiente de Mortalidade por faixa etária nos gêneros masculino e feminino em Diamantina (MG), entre 1998-2006.

Fonte: DATASUS (2007)

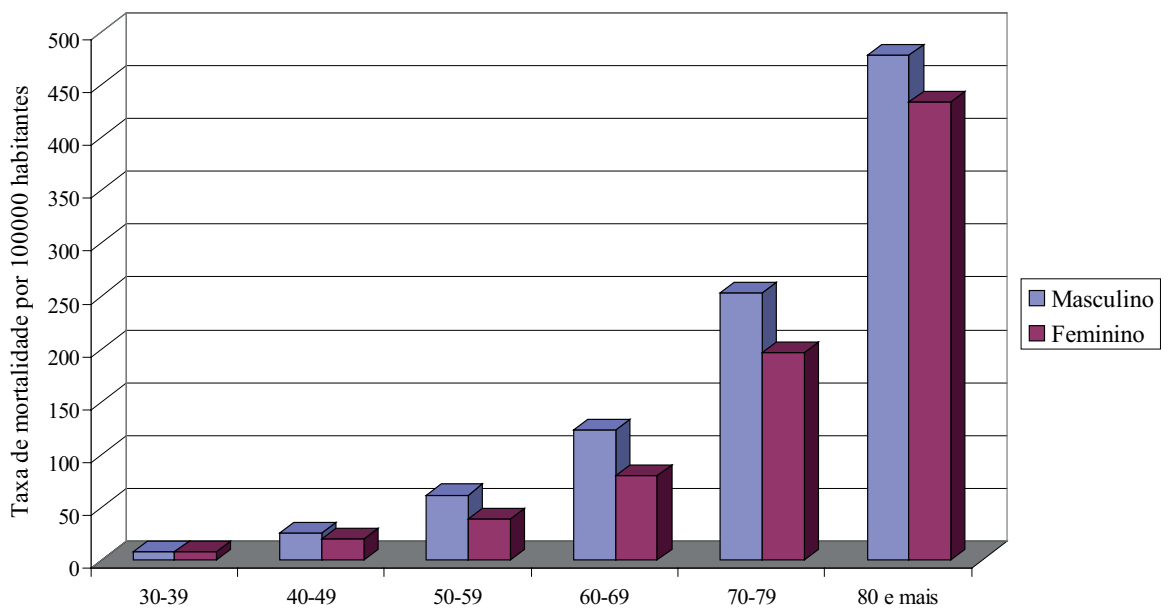

Gráfico 4b. Coeficiente de Mortalidade por faixa etária nos gêneros masculino e feminino em Minas Gerais, entre 1998-2006.

Fonte: DATASUS (2007)

Observou-se também a maior incidência de óbitos com o avançar da idade em ambos os gêneros, sendo maior em Diamantina (12.750,4/100.000 habitantes) do que em Minas Gerais $(9.088,2 / 100.000 \text { habitantes })^{14}$. Observaram aumento na incidência de DCV com o avançar da idade, de 100/100000 indivíduos com idade entre 45 e 54 anos para acima de 1800/100000 naqueles com idades superiores a 85 anos. Em um estudo ${ }^{14}$ realizado na regiáo metropolitana de Recife, observou-se que o número de óbitos referentes às DCV's são mais frequentes em indivíduos com idade superior a 75 anos, devido à dificuldade de locomoção das pessoas mais idosas para procurar assistência, particularmente se necessário locomover-se para outro município.

Embora os coeficientes de mortalidade de DCV por idade tenham aumentado com o avançar da idade, a Razão de Mortalidade Proporcional apresentou os seguintes valores: $30-39$ anos (16,67\%), 40-49 (17,12\%), 50-59 (18,03\%), 60-69 (17,44\%), 70-79 (17,65\%) e 80/ mais anos (12,44\%). No Brasil, as doenças do aparelho circulatório vêm determinando crescente número de óbitos entre adultos jovens, já a partir dos 20 anos assumindo o patamar de primeira causa de óbito na faixa dos 40 anos e predominando nas faixas etárias subsequentes ${ }^{2}$. As 
Tabela 1

Razão por Mortalidade Proporcional por faixa etária em Diamantina, entre 1998-2006

\begin{tabular}{cc}
\hline Faixa etária & RMP \\
\hline $30-39$ & $16,67 \%$ \\
$40-49$ & $17,12 \%$ \\
$50-59$ & $18,03 \%$ \\
$60-69$ & $17,44 \%$ \\
$70-79$ & $17,65 \%$ \\
80 e mais & $12,44 \%$ \\
\hline
\end{tabular}

Fonte: DATASUS (2007)

DCV's são responsáveis por aproximadamente 14,7\% dos óbitos na população acima de 30-40 anos ${ }^{14} \mathrm{e}$ incidências de $10 \%$ em pacientes com idade inferior a 55 anos e de 3,9\% em pacientes com idade inferior a 45 anos $^{7}$.

$\mathrm{Na}$ população idosa a etiologia e prevenção diferem da população jovem. Há nítida predominância da aterosclerose como causa da DCV. Nos jovens, prevalecem condiçóes hereditárias, más formaçóes e uso de drogas ilícitas ${ }^{3}$. Sabe-se também que o espectro de etiologia da DCV em jovens é maior que o observado em pacientes idosos, sugerindo que novos conhecimentos sobre a fisiopatogenia da DCV devem ser pesquisados ${ }^{7}$.

Diversas pesquisas epidemiológicas têm sido realizadas com objetivo de esclarecer a ocorrência da DCV devido ao considerável impacto individual e socioeconômico causado pela elevada taxa de morbi-mortalidade. $\mathrm{O}$ fato das doenças do aparelho circulatório atingir pessoas em idade produtiva tem um forte impacto econômico calculado por anos produtivos por vida perdidos ${ }^{2}$.

Mesmo quando a DCV não leva ao óbito, essa patologia causa com frequência deficiência parcial ou total do indivíduo, com graves repercussóes para ele, sua família e a sociedade1. Isso mostra que o investimento na sua prevenção é decisivo, não só para garantir a qualidade de vida aos indivíduos e seus familiares, mas, também para se evitarem gastos com a hospitalização, que se torna mais onerosa a cada dia, em razão do alto grau de sofisticação em que se encontra a medicina moderna.

O contexto no qual o indivíduo está inserido influencia a presença de óbitos por DCV em determinada região ${ }^{15}$. De acordo com os autores, o acesso às informaçôes de prevenção e aos serviços de saúde, particular- mente ao que se refere à assistência hospitalar, é reduzido nas regióes com condições socioeconômicas precárias. Salienta-se que, no município de Diamantina, não são desenvolvidos programas de prevenção às DCV's além de apresentar uma assistência hospitalar deficiente.

Em um estudo, investigou-se o perfil socioeconômico de 51 pacientes dos 82 cadastrados em todas Estratégias de Saúde da Família (ESF's) do município de Diamantina - MG, através de roteiro semi-estruturado. Dos indivíduos entrevistados, $49 \%$ e $41 \%$ eram analfabetos e possuíam uma renda familiar de um salário, respectivamente. Sugere-se que os indivíduos de baixo nível escolar e baixa renda salarial teriam menos acesso às informaçôes referentes aos fatores de prevenção, bem como condições financeiras insuficientes para manter uma dieta alimentar adequada. Salienta-se que tais fatores de risco não devem ser analisados individualmente, uma vez que a associação múltipla dos fatores etiológicos determinaria o modo de vida de um sujeito e sua consequente morbi-mortalidade ${ }^{16}$.

A implantação, como também melhorias das políticas de saúde na prevenção do acidente vascular cerebral (AVC) são essenciais no município de Diamantina ${ }^{17}$.

Devido ao elevado número de óbitos encontrados por DCV em Diamantina e a escassez de trabalhos relacionados aos seus coeficientes, salienta-se a necessidade de novos estudos para melhores esclarecimentos sobre a ocorrência da DCV na população adulta jovem e no que diz respeito aos picos de óbitos nos anos de 2000, 2003 e 2006. Além disso, há que considerar algumas limitaçôes do estudo tais como, possível subnotificação da causa de morte como sendo devido a DCV, considerando que os dados do DATASUS se baseiam no certificado de óbito, que muito frequentemente não consta a DCV como causa básica do óbito por erro no preenchimento.

\section{CONCLUSÃO}

O direito à saúde como dever do Estado é garantido à população brasileira por meio do Sistema Único de Saúde ${ }^{18}$. O SUS propóe o acesso universal, integral, igualitário e intersetorial às ações e serviços de promoção, proteção e recuperação da saúde, sendo as ações preventivas uma de suas prioridades ${ }^{19}$. Neste contexto, existe a relação entre o regimento do SUS e a atuação do fisioterapeuta. 
Apesar deste processo ainda estar em construção, observamos que o profissional fisioterapeuta vem adquirindo crescente participação nos serviços referentes à atenção primária. Isso porque suas funçôes e atribuiçôes são constituídas por conjunto de açóes de saúde, incluindo nessa esfera a prevenção, assim como o diagnóstico cinesiofuncional, tratamento, reabilitação e manutenção da saúde ${ }^{20}$, conforme o previsto no Conselho Federal de Fisioterapia e Terapia Ocupacional ${ }^{21}$. Neste panorama, salienta-se a necessidade da inserção de fisioterapeutas e outros profissionais da saúde, tais como terapeutas ocupacionais e fonoaudiólogos nas ESF's, a fim de garantir um melhor acesso aos serviços de atenção primária, secundária e terciária na atenção básica de saúde.

Dessa forma, são necessários outros estudos que objetivam identificar os fatores associados à mortalidade para que, num segundo momento, possa planejar e implementar políticas de saúde que visem reduzir essa mortalidade como também a morbidade possibilitando uma qualidade de vida para essas pessoas.

\section{REFERÊNCIAS}

1.Brasil, Ministério da Saúde. Programas e projetos: Doenças cardiovasculares. [Atualizado em: 27/2013; Acesso em: 27 de julho 2013]. Disponível em: http://dab.saude.gov.br/portaldab/doencas_cronicas.php.

2.Bocchi SCM, Ângelo M. Interação cuidador familiar-pessoa com AVC: autonomia compartilhada. Ciênci saúde Colet 2005;10:729-38.

http://dx.doi.org/10.1590/S1413-81232005000300029

3.Falcão IV, Carvalho EMF, Barreto KML, Lessa FJD, Leite VMM. Acidente vascular cerebral precoce: implicações para adultos em idade produtiva atendidos pelo sistema único de saúde. Rev Bras Saude Mater Infant 2004;4:95-102. http://dx.doi.org/10.1590/S1519-38292004000100009

4.Pires SL, Gagliard RJ, Gorzoni ML. Estudo das frequências dos principais fatores de risco para acidente vascular cerebral isquêmico em idosos. Arq Neuropsiquiatr 2004;62:844-51.

\section{http://dx.doi.org/10.1590/S0004-282X2004000500020}

5.Neto JE, Lotufo PA, Lólio CA. Tratamento da hipertensão e declínio da mortalidade por acidentes vasculares cerebrais. Rev Saúde Públ 1990;24:332-6. http://dx.doi.org/10.1590/S0034-89101990000400013

6.Feigin VL, Lawes CMM, Bennett DA, Anderson CS. Stroke epidemiology: a review of population-based studies of incidence, prevalence, and case-fatality in the late 20th century. Lancet 2003;2:43-53.

http://dx.doi.org/10.1016/S1474-4422(03)00266-7

7.Makiyama TY, Battisttella LR, Litvoc J, Martins LC. Estudo sobre a qualidade de vida de pacientes hemiplégicos por acidente vascular cerebral e de seus cuidadores. Acta Fisiátrica 2004;11:105-9.

8.Pittella JEH, Duarte JE. Prevalência e padrão de distribuição das doenças cerebrovasculares em 242 idosos, procedentes de um hospital geral, necropsiados em Belo Horizonte, Minas Gerais, no período de 1976 a 1997. Arq Neuropsiquiatr 2002;60:47-55.

http://dx.doi.org/10.1590/S0004-282X2002000100010

9.Zétola VHF, Novak EM, Camargo CHF, Júnior HC, Coral P, Muzzio JÁ, Iwamoto FM, Coleta MVD, Werneck LC. Acidente Vascular em pacientes jovens: Análise de 164 casos. Arq Neuropsiquiatr 2001;59:740-5.

http://dx.doi.org/10.1590/S0004-282X2001000500017

10.Brasil, Ministério da Saúde. DATASUS. [atualizado em: 07/2013; acessado em setembro de 2006 e 2007]. Disponível em: http://w3.datasus.gov.br/ datasus/datasus.php.

11.Radanovic M. Características do atendimento de pacientes com acidente vascular cerebral em hospital secundário. Arq Neuropsiquiatr 2000;58:99-106. http://dx.doi.org/10.1590/S0004-282X2000000100015

12.Lotufo PA, Bensenor IM. Stroke Mortality in São Paulo (1997-2003). Arq Neuropsiquiatr 2004;62:1008-11.

http://dx.doi.org/10.1590/S0004-282X2004000600014

13. Carritano CR, Luz PM, Pires MLE, Barbosa MTS, Batista KM. Análise da tendência da mortalidade por acidente vascular cerebral no Brasil no século XXI. Arquivo Bras. de Cardiologia 2012; [online].

14.Rodrigues JE, Sá MS, Alouche SR. Perfil dos pacientes acometidos por AVE tratados na clínica escola de fisioterapia da UMESP. Rev Neurocienc 2004; $12: 117-122$.

15.Carvalho EMF, Branco MAF. Perfil de mortalidade por doenças cardiovasculares na regiáo metropolitana de Recife, segundo o Sistema de Informação sobre Mortalidade (SIM). Info. Epidemiol. SUS 1996;4:61-71.

16.Leite HR, Nunes APN, Corrêa CL. Perfil dos Pacientes acometidos por Acidente Vascular encefálico cadastrados nas Estratégias de Saúde da Família do município de Diamantina, Minas Gerais. Fisioter Pesqui 2009;16:34-9.

17.Leite HR, Nunes APN, Corrêa CL. Study of Health-Quality of Life Among Chronic Stroke Survivors Living in Diamantina City-Brazil. Motor Control 2007; Suppl11:215-6.

18.Brasil, Sistema Único de Saúde. SUS: Aspectos gerais. [atualizado em: 07/2013; Acesso em: 28 maio 2007]. Disponível em: <http://www.sespa. pa.gov.br/SUS/sus/sus_aspgerais.htm>.

19.Brasil, Sistema Único de Saúde. SUS: O que é o SUS. [atualizado em: 07/2013; Acesso em: 28 jul. 2007]. Disponível em: <http://www.sespa.pa.gov. $\mathrm{br} / \mathrm{Sus} / \mathrm{sus} /$ sus_oquee.htm>.

20.Borges, AMP. A contribuição do fisioterapeuta para o Programa de Saúde da Família - uma revisão da literatura. Uniciencias 2010;14:69-82.

21.Conselho Federal de Fisioterapia e Terapia Ocupacional (COFFITO). [atualizado em: 07/2013; Acesso em: 15 maio 2008]. Disponível em: <http:// www.coffito.org.br/\#[page] showDynamic.php?page $=6 \&$ section $=6 \&$ pagina $=\mathrm{fi}$ sio_defin.> 